\title{
Avaliação vocal e cervicoescapular em militares instrumentistas de sopro
}

\section{Vocal and cervicoscapular evaluation in military wind instrumentalists}

\author{
Kelly Cristina Alves Silvérioํㅜ Eliane Cristina Pereira ${ }^{2}$, Laiza Maia Menoncin ${ }^{3}$, Cláudio Antonio Sorondo Dias ${ }^{4}$, \\ Celso Luis Gonçalves dos Santos Junior ${ }^{5}$, Paulo Prieto Y Schwartzman ${ }^{6}$
}

\begin{abstract}
RESUMO
Objetivo: Este estudo transversal e prospectivo objetivou avaliar qualidade vocal, respiração e região cervicoescapular em instrumentistas de sopro integrantes da Banda Musical do Exército. Métodos: Foram estudados 42 sujeitos do gênero masculino, militares, com idades entre 20 e 45 anos, divididos em: Grupo Experimental, com 30 instrumentistas de sopro, e Grupo Controle, com 12 instrumentistas de percussão. Todos passaram por entrevista; avaliação vocal - registro da voz e análise perceptivo-auditiva com uso da escala GRBASI; avaliação do tipo respiratório; avaliação fisioterapêutica da região cervicoescapular - inspeção visual dos perfis anterior, lateral e posterior, palpação dos músculos trapézio fibras-superiores, esternocleidomastoídeos, escalenos e base de occipital; e testes de mobilidade cervical e de encurtamentos musculares. Resultados: Não houve diferença entre Grupo Experimental e Controle quanto à presença de queixas vocais; os sujeitos do Grupo Experimental apresentaram mais sintomas laríngeos e relataram mais dor em músculo trapézio e região cervical quando comparados aos sujeitos do Controle. Os sujeitos do Grupo Experimental apresentaram maior alteração nos parâmetros vocais tensão e instabilidade do que os sujeitos do Grupo Controle. Constatou-se maior incidência da respiração costodiafragmático-abdominal no Grupo Experimental. Não houve diferença entre os grupos estudados quanto à postura, encurtamentos musculares e mobilidade cervical; porém o Grupo Experimental apresentou mais dor à palpação em músculo trapézio fibras superiores. Conclusão: Os militares instrumentistas de sopro apresentaram alterações vocais e da musculatura cervicoescapular e necessitam de ações de promoção à saúde vocal e postural.
\end{abstract}

Descritores: Avaliação/métodos; Qualidade da voz; Respiração; Postura; Militares; Música

\section{INTRODUÇÃO}

Entre as diversas atividades desenvolvidas pelos militares está a Banda Musical. Ela é constituída por instrumentistas

Trabalho realizado na Universidade Tuiuti do Paraná - UTP - Curitiba (PR), Brasil.

(1) Doutora, Professora do Departamento de Fonoaudiologia da Faculdade de Odontologia de Bauru da Universidade de São Paulo - USP - Bauru (SP), Brasil.

(2) Mestre, Fonoaudióloga da Prefeitura Municipal de Prudentópolis - Prudentópolis (PR), Brasil.

(3) Mestre, Docente dos cursos de Fisioterapia e Educação Física das Faculdades Integradas Espiritas - Curitiba (PR), Brasil.

(4) Mestre, Professor do Curso de Bacharelado em Música e Licenciatura em Música da Faculdade de Artes do Paraná - Curitiba (PR), Brasil.

(5) Mestre, Professor do Curso de Fonoaudiologia da Universidade Tuiuti do Paraná, Curitiba (PR), Brasil.

(6) Pós-graduando (Mestrado) em Distúrbios da Comunicação da Universidade Tuiuti do Paraná - UTP - Curitiba (PR), Brasil.

Endereço para correspondência: Kelly Cristina Alves Silvério. R. Maria José, 3-30, Centro, Bauru (SP), CEP: 17015-440. E-mail: kcsilver@terra.com.br

Recebido em: 24/9/2009; Aceito em: 22/3/2010 de sopro e de percussão e tem o compromisso com a música enquanto arte e a manutenção das tradições musicais militares.

A atividade de trabalho do músico exige concentração, atenção, memória, precisão, força, sincronia, criatividade, disciplina, dedicação, singularidade e cooperação, exigências essas direcionadas por uma hierarquia rígida que define as funções e os limites da ação de cada musicista ${ }^{(1)}$.

A prática árdua e a dedicação intensa sobre a técnica musical demandam um esforço físico e mental incomensurável aos musicistas, o que faz com que os mesmos exijam demasiadamente da sua estrutura física e mental. Todo este trabalho, parte do desempenho de excelente técnica e execução, reflete, ao corpo do instrumentista, possíveis e visíveis alterações posturais, encurtamentos e algias musculares devido à falta de consciência corporal. Além disso, os ângulos de atuação mecânica, ajustes finos de execução do instrumento e preocupações inconscientes de desempenho e aprimoramento da técnica prejudicam o ajuste ergonômico, por consequente ocasionando afecções musculoesqueléticas ${ }^{(2)}$. Desta forma, fatores ambientais e organizacionais do trabalho têm sido considerados causadores de risco para o desenvolvimento 
de distúrbios vocais, auditivos e musculoesqueléticos em músicos ${ }^{(1,3,4)}$.

Neste contexto, é crescente o número de instrumentistas que apresentam queixas de dor e distúrbios musculares relacionados à atividade de trabalho, por utilizarem elevada carga física, que acaba por sobrecarregar os membros superiores e exercer postura assimétrica e não ergonômica em relação aos instrumentos $^{(1)}$

Por outro lado, a execução de um instrumento musical envolve diferentes abordagens técnicas para obter os resultados desejados. No caso da produção do som, diferentes meios são usados, dependendo do instrumento. Com os instrumentos de sopro, a produção do som dá-se a partir do momento em que uma coluna de ar, dentro de um tubo, é colocada em movimento através da sua emissão por parte do executante ${ }^{(5)}$. Alguns estudos ${ }^{(6-8)}$ revelaram a participação ativa da laringe na execução do instrumento de sopro, sendo que o controle do fluxo aéreo e do sopro, aparentemente, estiveram relacionados à alternância entre constrição e abertura glótica. A respiração e suas respectivas pausas servem como elementos estruturais do desempenho musical, inclusive com conteúdo estético ${ }^{(9)}$.

Apesar de vários estudos abordarem problemas musculoesqueléticos em instrumentistas de banda musical ${ }^{(1,2,10,11)} \mathrm{e}$ apontarem a participação efetiva da laringe na execução de instrumentos de sopro ${ }^{(6,7)}$, é escasso o número de estudos que se propuseram a avaliar a voz, respiração e região cervicoescapular em instrumentistas de sopro.

Frente ao exposto, o objetivo deste estudo foi avaliar a qualidade vocal, a respiração e a região cervicoescapular em instrumentistas de sopro integrantes da Banda Musical do Exército.

\section{MÉTODOS}

Participaram deste estudo 42 indivíduos do gênero masculino, todos militares instrumentistas da banda musical do Exército de Curitiba (PR), subdivididos em dois grupos: Grupo Experimental (GE), composto de 30 indivíduos na faixa etária de 21 a 45 anos (média $=33,5$ anos), instrumentistas de sopro; e Grupo Controle (GC), que constou de 12 indivíduos na faixa etária de 25 a 54 anos (média $=37,1$ anos), da mesma banda, que executavam instrumentos de percussão.

Foram incluídos na pesquisa sujeitos do gênero masculino da banda do Exército que concordaram em participar de todas as avaliações. Foram excluídos da pesquisa aqueles que não participaram de uma das avaliações propostas pelo presente estudo e sujeitos do gênero feminino.

\section{Procedimento}

Após autorização dos superiores hierárquicos para a realização da pesquisa e a assinatura do Termo de Consentimento Livre e Esclarecido (CEP/UTP 00014/2008), os indivíduos do GE e do GC passaram por entrevista, avaliação vocal, respiratória e musculoesquelética da região cervicoescapular.

A entrevista constou da aplicação de um questionário com perguntas abertas e fechadas e investigou tempo de trabalho na função de músico, tipo de instrumento que executa, presença de queixa vocal (por meio da questão: "Você percebe algum problema em relação à sua voz?"), queixa de dor muscular (por meio da questão: "Você sente dor muscular em alguma região do corpo?" Se sim, o sujeito deveria assinalar o local, cujas opções constavam de uma lista), presença de sintomas laríngeos e vocais, hábitos vocais e de saúde e problemas respiratórios (por meio de uma lista de itens em que o sujeito deveria assinalar o que apresentava). Não foi investigada a graduação da dor muscular.

A avaliação vocal foi constituída de registro e análise perceptivo-auditiva das vozes.

Para o registro das vozes, utilizou-se o programa MultiDimensional Voice Program - MDVP - Modelo 5105 do software Multi-Speech Modelo 3700 - Kay Elemetrics, instalado em computador de marca Microtec, com placa de som da marca Sound Blaster, modelo Creative SB PCI 128 (Ensoniq ES 5880), microfone marca Plantronics áudio 90 - ultimate headset unidirecional, numa sala com tratamento acústico.

Foi solicitado ao indivíduo um trecho de fala espontânea e vogal sustentada. Para o registro da fala espontânea, cada sujeito respondeu às seguintes questões: "O que você acha de sua voz?" e "O que você faz no seu trabalho?" Para o registro da vogal sustentada, solicitou-se a emissão da vogal / $/$ / de maneira isolada, sustentada, e após inspiração profunda em pitch e loudness habituais, respeitando-se o tempo de emissão de cada sujeito. A escolha da vogal $/ \varepsilon /$ foi devida à postura neutra da língua na cavidade bucal, não necessitando de ajustes no trato vocal ${ }^{(12)}$.

Para a análise perceptivo-auditiva, os registros vocais foram randomizados e analisados por três fonoaudiólogos especialistas em voz, por consenso. Utilizou-se a escala GRBASI ${ }^{(13)}$ para análise da vogal $/ \varepsilon /$ e fala espontânea, que propiciou a avaliação dos parâmetros: grau de alteração global da voz $(\mathrm{G})$, rugosidade $(\mathrm{R})$, soprosidade $(\mathrm{B})$, astenia $(\mathrm{A})$, tensão $(\mathrm{S})$ e instabilidade (I) em graus que variaram de zero a três, onde zero significou ausência de alteração, um indicou alteração de grau leve, dois - alteração de grau moderado e três - alteração de grau extremo.

Para a avaliação da respiração foi solicitado que cada sujeito executasse uma inspiração e expiração profundas e foram observados os movimentos dos músculos intercostais, diafragmáticos e abdominais, na posição deitado, para a classificação da respiração em clavicular, torácica ou costodiafragmáticoabdominal ${ }^{(13)}$. Esta avaliação foi realizada por fonoaudiólogo e por fisioterapeuta, por consenso.

A avaliação musculoesquelética da região cervicoescapular foi realizada individualmente, por fisioterapeuta, pesquisadora deste estudo. Constou de inspeção visual da postura, exame de palpação da cintura escapular, teste de encurtamento muscular e de mobilidade cervical ${ }^{(14)}$. Na inspeção visual da postura foram visualizados os perfis anterior, lateral e posterior. Foi realizada a inspeção anterior, observando-se o eixo cervical, se havia inclinação lateral ou rotação cervical. Já no perfil lateral, foi observado se havia anteriorização cervical, bem como hiperlordose ou retificação cervical. Na inspeção posterior foi verificada a presença ou não de rotação e inclinação cervical.

A segunda parte da avaliação musculoesquelética consistiu em exame de palpação dos músculos trapézio - fibras superiores, esternocleidomastóideos, escalenos e base de occipital 
(linha nucal inferior), com intuito de identificar presença ou ausência de dor.

Posteriormente, seguiu-se com o teste de encurtamento muscular: investigação sobre presença ou ausência de encurtamentos musculares em músculo trapézio - fibras superiores e esternocleidomastóideo.

Para o teste de mobilidade cervical solicitou-se ao sujeito que realizasse a flexão, extensão, rotação e inclinação lateral da coluna cervical de maneira ativa e foram investigados presença ou ausência de alteração nos movimentos solicitados.

\section{Análise dos dados}

Os dados foram organizados e tratados estatisticamente. Foi aplicado o teste de diferença de proporções (nível de significância 0,05) a fim de comparar os dados da entrevista, a respiração e a avaliação musculoesquelética do GE e do GC. Para a comparação dos dados da avaliação perceptivo-auditiva entre os grupos foram utilizados os testes de Fischer e Quiquadrado (nível de significância 0,05).

\section{RESULTADOS}

\section{Entrevista}

Os dados da entrevista revelaram que a média de tempo de trabalho dos instrumentistas do GE na função de músico foi de 18,1 anos, e do GC 12,8 anos.

Embora não tenha sido observada diferença entre os grupos estudados quanto à presença de queixas vocais foi possível observar que rouquidão e falhas na voz foram as queixas mais relatadas pelos instrumentistas de sopro (GE). Por outro lado, os sujeitos do GE apresentaram mais sintomas laríngeos, quando comparados aos sujeitos do GC. Os sintomas mais relatados pelo GE foram: dor de garganta, pigarro e garganta seca após tocar os instrumentos (Tabela 1).

A maioria dos sujeitos do GE e do GC apresentou algum tipo de queixa de dor musculoesquelética. Entretanto, houve diferença estatística entre os grupos estudados em relação à presença de dor em músculo trapézio e região cervical, mais relatada pelos sujeitos do GE (Tabela 1).

Quanto à presença de hábitos vocais e de saúde inadequados, o GE apresentou maior incidência do que o GC, quanto a fazer uso da voz sob competição sonora, ingerir bebidas geladas e ingerir álcool (Tabela 1).

Quanto à presença de problemas respiratórios, houve uma tendência à diferença entre os grupos estudados quando considerado o total de sujeitos do GE e do GC ao relatarem rinite alérgica e respiração bucal (Tabela 1).

\section{Qualidade vocal e respiração}

$\mathrm{Na}$ análise perceptivo-auditiva da vogal / $/$ /, por meio da escala GRBASI foi possível observar que os sujeitos do GE

Tabela 1. Incidência de queixas vocais, sintomas laríngeos, hábitos de saúde e vocais inadequados e problemas respiratórios nos grupos Experimental e Controle

\begin{tabular}{|c|c|c|c|c|c|c|}
\hline \multirow{2}{*}{ Dados da entrevista } & \multirow{2}{*}{ Parâmetro avaliado } & \multicolumn{2}{|c|}{ GE } & \multicolumn{2}{|c|}{$\mathrm{GC}$} & \multirow{2}{*}{ Valor de $p$} \\
\hline & & $\mathrm{N}$ & $\%$ & $\mathrm{~N}$ & $\%$ & \\
\hline \multirow{3}{*}{ Queixas vocais } & Rouquidão & 3 & 10 & 2 & 16,6 & 0,553 \\
\hline & Falhas na voz & 2 & 6,66 & 0 & 0 & 0,365 \\
\hline & Total queixas vocais & 5 & 16,6 & 2 & 16,6 & 1,000 \\
\hline \multirow{4}{*}{$\begin{array}{l}\text { Queixas de dor } \\
\text { musculoesquelética }\end{array}$} & Região lombar & 3 & 10 & 4 & 33,3 & 0,075 \\
\hline & Músculo trapézio fibras superiores e região cervical & 23 & 76,6 & 5 & 41,6 & $0,0036^{*}$ \\
\hline & Região dorsal & 2 & 6,6 & 0 & 0 & 0,365 \\
\hline & Total queixas de dor musculoesquelética & 28 & 93,3 & 9 & 75 & 0,106 \\
\hline \multirow{4}{*}{ Sintomas laríngeos } & Dor de garganta & 5 & 16,6 & 1 & 8,3 & 0,475 \\
\hline & Pigarro & 5 & 16,6 & 0 & 0 & 0,141 \\
\hline & Garganta seca após tocar instrumentos & 4 & 13,3 & 0 & 0 & 0,192 \\
\hline & Total sintomas laríngeos & 14 & 46,6 & 1 & 8,3 & $0,024^{*}$ \\
\hline \multirow{6}{*}{$\begin{array}{l}\text { Hábitos vocais e de saúde } \\
\text { inadequados }\end{array}$} & Uso da voz sob competição sonora & 15 & 50 & 1 & 8,3 & $0,016^{*}$ \\
\hline & Gritar & 7 & 23,3 & 0 & 0 & 0,075 \\
\hline & Ingerir bebidas geladas & 10 & 33,3 & 0 & 0 & $0,027^{*}$ \\
\hline & Uso de tabaco & 8 & 26,6 & 1 & 8,3 & 0,198 \\
\hline & Uso de álcool & 14 & 46,6 & 0 & 0 & $0,006^{*}$ \\
\hline & Total hábitos inadequados & 25 & 83,3 & 2 & 16,6 & $0,000^{*}$ \\
\hline \multirow{3}{*}{ Problemas respiratórios } & Rinite alérgica & 8 & 26,6 & 2 & 16,6 & 0,495 \\
\hline & Respiração bucal & 7 & 23,3 & 0 & 0 & 0,075 \\
\hline & Total de problemas respiratórios & 15 & 50 & 2 & 16,6 & 0,053 \\
\hline
\end{tabular}

Teste de Diferença de Proporções

* Valores significativos $(\mathrm{p} \leq 0,05)$ 
apresentaram maior alteração nos parâmetros vocais tensão e instabilidade do que os sujeitos do GC (Tabela 2). Na fala espontânea, não houve diferença em nenhum parâmetro vocal analisado (Tabela 3).

$\mathrm{Na}$ avaliação respiratória foi possível observar que não houve diferença entre os grupos estudados quanto ao tipo de respiração clavicular e torácica. Entretanto, constatouse diferença entre os grupos quanto ao tipo de respiração costodiafragmático-abdominal, com maior incidência desta respiração no GE.

\section{Avaliação da região cervicoescapular}

A avaliação postural não evidenciou nenhuma diferença entre os grupos estudados nas vistas: anterior, posterior e lateral, sendo que rotação cervical, inclinação lateral e anteriorização cervical foram alterações observadas em ambos os grupos (Tabela 4).
O exame de palpação de musculatura de trapézio (fibras superiores), esternocleidomastoídeos, escalenos e base de occipital (linha nucal inferior), evidenciou presença de pontos de dor em músculo trapézio - fibras superiores, com diferença significativa entre os grupos $(\mathrm{p}=0,011)$ (Tabela 5).

O teste de encurtamento muscular revelou que não houve diferença entre os grupos estudados. Porém, foi possível observar uma tendência à significância quanto à presença de encurtamento muscular em músculo trapézio, maior nos sujeitos do GE quando comparados aos sujeitos do GC ( $\mathrm{p}=0,071)$ (Tabela 5).

O teste de mobilidade cervical revelou que não houve diferença quanto ao comprometimento da musculatura cervical dos grupos estudados, porém a inclinação cervical foi o parâmetro mais comprometido em ambos os grupos: 96,6\% do GE e 83,3\% do GC (Tabela 5).

Tabela 2. Resultados da análise perceptivo-auditiva da vogal /ع/, com uso da escala GRBASI dos Grupos Experimental e Controle

\begin{tabular}{|c|c|c|c|c|c|c|}
\hline \multirow[t]{2}{*}{ Variável } & \multirow[t]{2}{*}{ Grupo } & \multicolumn{3}{|c|}{ Resultados } & \multirow[t]{2}{*}{ Teste } & \multirow[t]{2}{*}{ Valor de $p$} \\
\hline & & 0 & 1 & 2 & & \\
\hline \multirow[t]{2}{*}{ G } & Experimental & 6 & 44 & 40 & Qui-quadrado & 0,8574 \\
\hline & Controle & 3 & 17 & 16 & & \\
\hline \multirow[t]{2}{*}{$\mathrm{R}$} & Experimental & 22 & 43 & 25 & Qui-quadrado & 0,2887 \\
\hline & Controle & 6 & 16 & 14 & & \\
\hline \multirow[t]{2}{*}{ B } & Experimental & 72 & 18 & 0 & Qui-quadrado & 0,7020 \\
\hline & Controle & 27 & 8 & 1 & & \\
\hline \multirow[t]{2}{*}{ A } & Experimental & 90 & - & - & Não se aplica & - \\
\hline & Controle & 36 & - & - & & \\
\hline \multirow[t]{2}{*}{ S } & Experimental & 24 & 55 & 11 & Qui-quadrado & $0,0000^{*}$ \\
\hline & Controle & 36 & - & - & & \\
\hline \multirow[t]{2}{*}{ I } & Experimental & 0 & 64 & 26 & Qui-quadrado & $0,0000^{*}$ \\
\hline & Controle & 16 & 17 & 3 & & \\
\hline
\end{tabular}

Nota: Para possibilitar o teste para a variável Soprosidade (B), considerou-se duas categorias de resultados 0 e $1+2$

* Valores significativos $(\mathrm{p} \leq 0,05)$

Legenda: $\mathrm{G}$ = grau de alteração da voz; $\mathrm{R}$ = rugosidade; $\mathrm{B}=$ soprosidade; $\mathrm{A}$ = astenia; $\mathrm{S}=$ tensão; I = instabilidade

Tabela 3. Resultados da análise perceptivo-auditiva da fala espontânea com uso da escala GRBAS dos Grupos Experimental e Controle

\begin{tabular}{|c|c|c|c|c|c|c|}
\hline \multirow[t]{2}{*}{ Variável } & \multirow[t]{2}{*}{ Grupo } & \multicolumn{3}{|c|}{ Resultados } & \multirow[t]{2}{*}{ Teste } & \multirow[t]{2}{*}{ Valor de $p$} \\
\hline & & 0 & 1 & 2 & & \\
\hline \multirow[t]{2}{*}{ G } & Experimental & 7 & 45 & 38 & Qui-quadrado & 0,0560 \\
\hline & Controle & 5 & 24 & 7 & & \\
\hline \multirow[t]{2}{*}{$\mathrm{R}$} & Experimental & 13 & 50 & 27 & Qui-quadrado & 0,2302 \\
\hline & Controle & 9 & 19 & 8 & & \\
\hline \multirow[t]{2}{*}{ B } & Experimental & 81 & 9 & - & Fisher & 1,0000 \\
\hline & Controle & 33 & 3 & - & & \\
\hline \multirow[t]{2}{*}{ A } & Experimental & 90 & - & - & Não se aplica & - \\
\hline & Controle & 36 & - & - & & \\
\hline \multirow[t]{2}{*}{ S } & Experimental & 26 & 48 & 16 & Qui-quadrado & 0,0606 \\
\hline & Controle & 18 & 14 & 4 & & \\
\hline
\end{tabular}

Legenda: $\mathrm{G}$ = grau de alteração da voz; $\mathrm{R}$ = rugosidade; $\mathrm{B}=$ soprosidade; $\mathrm{A}=$ astenia; $\mathrm{S}=$ tensão 
Tabela 4. Resultados da inspeção visual anterior, posterior e lateral na avaliação postural dos Grupos Experimental e Controle

\begin{tabular}{|c|c|c|c|c|c|c|c|c|c|c|c|c|c|c|c|}
\hline \multirow[t]{3}{*}{ Avaliação postural } & \multicolumn{5}{|c|}{ Vista anterior } & \multicolumn{5}{|c|}{ Vista posterior } & \multicolumn{5}{|c|}{ Vista lateral } \\
\hline & \multicolumn{2}{|c|}{ GE } & \multicolumn{2}{|c|}{$\mathrm{GC}$} & \multirow[b]{2}{*}{$\begin{array}{l}\text { Valor } \\
\text { de } p\end{array}$} & \multicolumn{2}{|c|}{ GE } & \multicolumn{2}{|c|}{$\mathrm{GC}$} & \multirow[b]{2}{*}{$\begin{array}{l}\text { Valor } \\
\text { de } p\end{array}$} & \multicolumn{2}{|c|}{ GE } & \multicolumn{2}{|c|}{ GC } & \multirow[b]{2}{*}{$\begin{array}{l}\text { Valor } \\
\text { de } p\end{array}$} \\
\hline & $\mathrm{N}$ & $\%$ & $\mathrm{~N}$ & $\%$ & & $\mathrm{~N}$ & $\%$ & $\mathrm{~N}$ & $\%$ & & $\mathrm{~N}$ & $\%$ & $\mathrm{~N}$ & $\%$ & \\
\hline Rotação cervical & 14 & 46,6 & 8 & 66,6 & 0,299 & 14 & 46,6 & 8 & 66,6 & 0,300 & 0 & 0 & 0 & 0 & - \\
\hline Inclinação lateral & 15 & 50 & 4 & 33,3 & 0,383 & 15 & 50 & 4 & 33,3 & 0,383 & 0 & 0 & 0 & 0 & - \\
\hline Retificação cervical & 0 & 0 & 0 & 0 & - & 0 & 0 & 0 & 0 & 0 & 4 & 13,3 & 0 & 0 & 0,192 \\
\hline Anteriorização cervical & 0 & 0 & 0 & 0 & - & 0 & 0 & 0 & 0 & 0 & 18 & 60 & 8 & 66,6 & 0,723 \\
\hline Hiperlordose cervical & 0 & 0 & 0 & 0 & - & 0 & 0 & 0 & 0 & 0 & 7 & 23,3 & 4 & 33,3 & 0,550 \\
\hline Normal & 1 & 3,33 & 0 & 0 & 0,528 & 1 & 3,33 & 0 & 0 & 0,528 & 1 & 3,3 & 0 & 0 & 0,528 \\
\hline
\end{tabular}

Teste de diferença de proporções $(p \leq 0,05)$

Legenda: $\mathrm{GE}$ = grupo experimental; $\mathrm{GC}$ = grupo controle

Tabela 5. Identificação de pontos de dor à palpação, resultados do teste de encurtamento muscular e exame de mobilidade cervical dos Grupos Experimental e Controle

\begin{tabular}{|c|c|c|c|c|c|c|}
\hline \multirow{2}{*}{ Teste fisioterápico } & \multirow{2}{*}{ Parâmetro avaliado } & \multicolumn{2}{|c|}{ GE } & \multicolumn{2}{|c|}{ GC } & \multirow[t]{2}{*}{ Valor de $\mathrm{p}$} \\
\hline & & $\mathrm{N}$ & $\%$ & $\mathrm{~N}$ & $\%$ & \\
\hline \multirow{3}{*}{ Palpação } & Dor em músculo TFS & 30 & 100 & 9 & 75 & ${ }^{\star} 0,011$ \\
\hline & Dor em músculo ECM & 0 & 0 & 0 & 0 & - \\
\hline & Dor em base de occípto & 0 & 0 & 0 & 0 & - \\
\hline \multirow{4}{*}{ Teste de encurtamento muscular } & Músculo TFS com encurtamento & 30 & 100 & 10 & 83,3 & 0,071 \\
\hline & Músculo TFS sem encurtamento & 0 & 0 & 2 & 16,6 & - \\
\hline & Músculo ECM com encurtamento & 23 & 76,6 & 9 & 75 & 0,945 \\
\hline & Músculo ECM sem encurtamento & 7 & 23,3 & 3 & 25 & 0,945 \\
\hline \multirow{4}{*}{ Mobilidade cervical } & Flexão cervical comprometida & 1 & 3,3 & 2 & 16,6 & 0,1373 \\
\hline & Extensão cervical comprometida & 2 & 6,6 & 0 & 0 & 0,3674 \\
\hline & Inclinação cervical comprometida & 29 & 96,6 & 10 & 83,3 & 0,1398 \\
\hline & Rotação cervical comprometida & 5 & 16,6 & 3 & 25 & 0,5343 \\
\hline
\end{tabular}

Teste de diferença de proporções

* Valores significativos $(\mathrm{p} \leq 0,05)$

Legenda: TFS = Trapézio fibras superiores; $\mathrm{ECM}=$ Esternocleidomastóideo; GE = grupo experimental; GC = grupo controle

\section{DISCUSSÃO}

Quanto às queixas vocais (rouquidão e falhas na voz) apresentadas pelos indivíduos, alguns estudos afirmaram que estas queixas são fatores predisponentes da disfonia ocupacional, que advém do uso inadequado que os sujeitos fazem da voz ${ }^{(15-17)}$, embora apenas $16,6 \%$ dos sujeitos instrumentistas de sopro tenham relatado queixas vocais.

Os sintomas laríngeos dor de garganta, pigarro e garganta seca após tocar os instrumentos indicam tensão e falta de hidratação laríngea, as quais podem acompanhar ou não as mudanças na $\mathrm{voz}^{(18)}$. Os resultados deste estudo estão de acordo com pesquisa ${ }^{(8)}$ que encontrou, entre músicos instrumentistas de sopro, o pigarro e a disfonia após intenso uso do instrumento. A ausência de relação, por parte dos sujeitos, de sintomas laríngeos com problemas vocais torna evidente a falta de consciência dos sinais da disfonia e o desconhecimento em relação à saúde vocal ${ }^{(19,20)}$. Os dados encontrados no presente estudo refletem tal realidade, pois fazer uso da voz sob competição sonora, ingerir bebidas geladas e fazer uso de álcool foram hábitos mais evidentes nos sujeitos do GE, que já possuem atividade profissional que requer a necessidade de uso intenso da laringe.

Estes dados apontam para a necessidade de um programa de preservação vocal que contenha orientações aos músicos instrumentistas de sopro, visando prevenir possíveis lesões laríngeas que pioram a situação da fala e do desempenho musical.

Em relação às queixas musculoesqueléticas, os resultados deste estudo mostraram valores superiores aos encontrados na literatura. Alguns estudos revelaram prevalência de queixas musculoesqueléticas referentes à prática instrumental que variaram de $26 \%$ a $87 \%$ entre os músicos ${ }^{(1,2,10)}$, sendo as queixas mais comuns: dor, rigidez, fadiga e tensão muscular. Aparentemente, os instrumentos apresentam características que os tornam fontes preferenciais para o surgimento de certas alterações; porém, não existe uma classificação de instrumentos que revele frequência absoluta de queixas ${ }^{(2)}$. Mais recentemente, um estudo ${ }^{(1)}$ encontrou prevalência de queixas musculoesqueléticas, que varia de 55\% a $86 \%$ em músicos profissionais de orquestras, sendo essa uma porcentagem elevada quando comparada a outras profissões que correlacionaram esses tipos de afecções no trabalho. 
$\mathrm{Na}$ análise perceptivo-auditiva, os dados de tensão e instabilidade vocais significativamente mais alterados nos sujeitos do GE na análise da vogal $/ \varepsilon /$, revelam que os sujeitos instrumentistas de sopro podem desenvolver um quadro de disfonia, pois estes parâmetros alterados podem indicar uso de laringe sob esforço. Estes dados podem ser explicados por estudos que se propuseram a avaliar a configuração glótica em instrumentistas de sopro $^{(6-8)}$ e encontraram uma grande participação da laringe na execução do instrumento de sopro.

Segundo estudo ${ }^{(8)}$ que avaliou a configuração glótica em instrumentistas de sopro, verificando a participação do trato vocal na execução dos instrumentos, por meio de nasofibrolaringoscopia, todos os sons musicais foram produzidos durante a adução das pregas vocais e o controle do fluxo aéreo e do sopro, aparentemente, esteve relacionado à alternância entre constrição e abertura glótica. Desta forma, este mesmo estudo $^{(8)}$ verificou que há adução das pregas vocais durante o sopro utilizado para tocar o instrumento, o que pode causar maior dificuldade técnica relacionada à tensão glótica (constrição látero-lateral) e supraglótica durante a execução do instrumento. Contraria, portanto, a noção histórica de que as pregas vocais não atuariam diretamente na produção do som do instrumento, sendo o esforço feito apenas pela musculatura respiratória e oromandibular, com a laringe aberta para permitir a passagem do $\operatorname{ar}^{(21,22)}$.

O sopro sonorizado, relacionado às técnicas utilizadas e à frequência de execução do instrumento, pode tornar-se um abuso vocal, uma vez que o trato vocal é usado intensamente, o que pode gerar alterações na configuração glótica, interferindo na produção sonora musical final e podendo causar, isolado ou associado a demais fatores, uma disfonia ${ }^{(8)}$.

Outros autores ${ }^{(6)}$ avaliaram os movimentos da laringe e hipofaringe durante a execução do saxofone e observaram fechamento velofaríngeo, posição laríngea constantemente baixa, vestíbulo laríngeo ligeiramente estreitado, adução das pregas vocais durante a execução de um tom inteiro, pregas vocais em posição paramediana no piano-forte-glissando, sendo que a laringe parece participar efetivamente regulando a corrente de ar. Da mesma forma, outro estudo ${ }^{(7)}$ constatou intenso fechamento velofaríngeo, bem como deslocamento das pregas vocais durante o vibrato na execução do instrumento de sopro.

Neste contexto, talvez orientações sobre saúde vocal e desenvolvimento de técnicas de aquecimento vocal antes da execução do instrumento de sopro possam ser realizados com os instrumentistas de sopro, a fim de prevenir problemas vocais.

A avaliação respiratória evidenciou maior incidência do tipo de respiração costodiafragmático-abdominal no GE, o que é esperado no instrumentista de sopro, pois este tipo de respiração caracteriza-se por uma expansão harmônica de toda a caixa torácica, sem excessos na região superior ou inferior ${ }^{(13)}$. Uma vez que os instrumentistas de sopro utilizam com mais intensidade a musculatura abdominal para buscar suporte muscular ao executar o instrumento, seria esperado que os mesmos apresentassem um tipo de respiração mais completa do que músicos instrumentistas de percussão que fizeram parte, nesta pesquisa, do GC.

Os resultados deste estudo concordam com os achados de uma pesquisa ${ }^{(23)}$ que objetivou comparar a respiração nas situações de fonação e durante a execução de instrumento de sopro e constatou que todos os indivíduos referiam que utilizavam a mesma respiração utilizada pelos cantores, porém apresentaram respiração costodiafragmática durante a execução do instrumento e costal superior para a fonação. Entretanto, é importante ressaltar que se o tipo de respiração costodiafragmático-abdominal estiver presente na fala habitual, a pressão da coluna de ar infraglótica será tão grande que um estado glótico hipertônico poderá ser instalado, na tentativa de impedir uma grande saída de ar, exigindo um trabalho intenso da musculatura respiratória para manter a caixa torácica alargada e elevada, o que pode gerar uma fonação tensa e comprimida, levando à fadiga vocal ${ }^{(13)}$. No presente estudo, os sujeitos foram avaliados solicitando-se uma inspiração e expiração profunda, deitados, o que facilita a percepção do tipo de respiração por parte do avaliador, porém, não é possível afirmar que o uso deste tipo de respiração, nos sujeitos do presente estudo, seja realizado na execução do instrumento ou mesmo durante a fala habitual.

Quanto à avaliação da região cervicoescapular, foi possível observar que rotação cervical, inclinação lateral e anteriorização cervical foram alterações observadas em ambos os grupos. Portanto, tanto os instrumentistas de sopro como de percussão, participantes deste estudo, apresentaram importantes alterações posturais.

Não foram encontrados estudos que tenham se proposto a discutir sobre alterações cervicoescapulares em musicistas; porém acredita-se que essas alterações posturais em região cervicoescapular sejam decorrentes da falta de consciência corporal, manutenção da postura ao praticar o instrumento, excesso de tempo na prática da ação, falha na ergonomia em relação à posição dos instrumentos e do músico, bem como falta de alongamento e preparo muscular, o que pode acarretar dores localizadas e desequilíbrios musculares.

Os pontos de dor à palpação encontrados em músculo trapézio - fibras superiores em todos os sujeitos do GE e na maioria dos sujeitos do GC é decorrente de alteração postural, o que causa microtraumatismos na musculatura, desenvolvendo nódulos hiperirritáveis resultantes de posição de tensão muscular, assimetrias e desequilíbrios posturais ou esqueléticos, por manter a posição de trabalho por tempos longos ${ }^{(24)}$. Da mesma forma, a presença de encurtamento muscular nos músculos esternocleidomastóideo e trapézio - fibras superiores denota permanência de uma mesma postura por tempo prolongado, falha ergonômica e falta de consciência corporal ${ }^{(24)}$.

Este resultado deve-se à postura inadequada de posição de cabeça, especialmente nos musicistas de sopro, por manterem a posição da cabeça em rotação, provocando uma importante retração na musculatura rotadora de pescoço. Este mesmo sinal também está presente em outros instrumentistas devido à posição de partitura. Acredita-se que os desalinhamentos posturais são as ações musculares inúteis mais frequentes e problemáticas entre pianistas ${ }^{(10)}$.

Desta forma, a prática de executar algum tipo de instrumento pode facilitar o desenvolvimento de posturas incorretas ou erros posturais por meio da solicitação exacerbada de um dos membros, ou facilitar o reequilíbrio postural pelo reconheci- 
mento corporal, buscando o equilíbrio homeostático corporal, evitando assim, alterações musculoesqueléticas ${ }^{(25)}$.

Os dados obtidos neste estudo demonstram a necessidade de desenvolvimento de um programa de saúde vocal, com orientações e conscientização a respeito da mesma, bem como preparo da laringe e da voz antes da execução do instrumento de sopro, a fim de evitar problemas vocais futuros. Considerando-se alteração postural um problema de saúde que pode provocar incapacidade profissional definitiva ou temporária ${ }^{(25)}$, é importante o desenvolvimento de ações de promoção à saúde que contenham orientações aos músicos quanto à importância de uma boa postura não só durante a execução de instrumentos, mas em todas as atividades a serem desenvolvidas.

\section{CONCLUSÃO}

Os instrumentistas de sopro da banda musical do Exército apresentaram queixas de rouquidão e falhas na voz e mais sintomas laríngeos quando comparados aos sujeitos do grupo controle, sendo que a queixa de dor musculoesquelética esteve presente em ambos os grupos.

Os instrumentistas de sopro apresentaram maior incidência de hábitos vocais e de saúde inadequados do que os sujeitos do grupo controle, sendo que fazer uso da voz sob competição sonora, consumir bebidas geladas e álcool foram os hábitos prejudiciais mais relatados.

Ambos os grupos apresentaram alterações na qualidade vocal, com alteração nos parâmetros de tensão e instabilidade nos instrumentistas de sopro. Todos os sujeitos estudados apresentaram importantes alterações posturais, encurtamentos musculares e alterações de mobilidade cervical. A anteriorização cervical, com pontos de dor à palpação em músculos trapézio - fibras superiores foi mais incidente nos instrumentistas de sopro. Estes dados refletem a necessidade de ações de promoção à saúde vocal e postural em músicos.

\begin{abstract}
Purpose: This cross-sectional prospective study had the aim to evaluate vocal quality, breathing and the cervicoscapular region in wind instrumentalists of the Army Band. Methods: Forty-two male military subjects with ages ranging from 20 to 45 years were divided into: Experimental Group, with 30 wind instrumentalists, and Control Group, with 12 percussionists. All subjects underwent an interview, vocal evaluation - voice record and auditory-perceptual analysis using the GRBASI scale, respiratory type evaluation, physical therapy evaluation of the cervicoscapular region - visual examination of anterior, lateral and posterior profiles, palpation of the trapezius, sternocleidomastoid, scalene and base of occipital muscles, and cervical mobility and muscle shortenings tests. Results: No difference was found between Experimental and Control groups regarding the presence of vocal complaints; subjects from the Experimental Group had more laryngeal symptoms and reported more pain in the trapezius muscle and the cervical region, when compared to control subjects. They also had greater alterations in vocal tension and instability parameters than subjects from the Control Group. There was a higher incidence of costodiaphragmatic-abdominal breathing among the Experimental Group subjects. There was no difference between the groups regarding posture, muscle shortenings, and cervical mobility; however, the Experimental Group presented more pain during the palpation of the trapezius muscle. Conclusion: The military wind instrumentalists presented vocal and cervicoscapular alterations, and require actions to promote vocal and postural health.
\end{abstract}

Keywords: Evaluation/methods; Voice quality; Breathing; Posture; Muscle; Military personnel; Music

\section{REFERÊNCIAS}

1. Frank A, Mühlen CAV. Queixas musculoesqueléticas em músicos: prevalência e fatores de risco. Rev Bras Reumatol. 2007;47(3):188-96.

2. Middlestadt SE, Fishbein M. The prevalence of severe musculoskeletal problems among male and female symphony orchestra string players. Med Probl Perform Art. 1989; 4(1):41-8.

3. Williams NR. Occupational groups at risk of voice disorders: a review of the literature. Occup Med (Lond). 2003; 53(7):456-60.

4. Ferreira LP, Akutsu CM, Luciano P, Viviano NDAG. Condições de produção vocal de teleoperadores: correlações entre questões de saúde, hábitos e sintomas vocais. Rev Soc Bras Fonoaudiol. 2008;13(4):307-15.

5. Methfessel G. [Myofunction in players of wind instruments]. Dtsch Zahnarztl Z. 1990;45(7 Spec No):48-50. German.

6. Weikert M, Schlömicher-Thier J. Laryngeal movements in saxophone playing: video-endoscopic investigations with saxophone players. A pilot study. J Voice. 1999;13(2):265-73.

7. Kahane JC, Beckford NS, Chorna LB, Teachey JC, McClelland DK. Videofluoroscopic and laryngoscopic evaluation of the upper airway and larynx of professional bassoon players. J Voice. 2006;20(2):297-307.
8. Eckley CA. Configuração glótica em tocadores de instrumento de sopro. Rev Bras Otorrinolaringol. 2006;72(1):45-7.

9. Davies J, Mangion S. Predictors of pain and other musculoskeletal symptoms among professional instrumental musicians: elucidating specific effects. Med Probl Perform Art. 2002;17(4):155-65.

10. Shields N, Dockrell S. The prevalence of injuries among pianists in music schools in Ireland. Med Probl Perform Art. 2000;15(4):155-60.

11. Kaneko Y, Lianza S, Dawson WJ. Pain as a incapacitating factor in symphony orchestra musicians in Sao Paulo, Brazil. Med Probl Perform Art. 2005;20(4):168-74.

12. Rezende A, Guedes AP, Almeida C, Gadel M, Borrego MC, Azevedo R, Gonçalves MI, Behlau M, Pontes P. Constrição anteroposterior do vestíbulo laríngeo em indivíduos normais durante a fonação sustentada. Rev Soc Bras Fonoaudiol. 1998;2(4):22-8.

13. Behlau M, organizadora. Voz: o livro do especialista. Rio de Janeiro: Revinter; 2001. Vol. 1.

14. Knoplich J. Enfermidades da coluna vertebral. 2a ed. São Paulo: Panamed; 1986. 
15. Nix J, Svec JG, Laukkanen AM, Titze IR. Protocol challenges for onthe-job voice dosimetry of teachers in the United States and Finland. J Voice. 2007;21(4):385-96.

16. Hamdan AL, Sibai AM, Srour ZM, Sabra OA, Deeb RA. Voice disorders in teachers. The role of family physicians. Saudi Med J. 2007;28(3):4228.

17. Niebudeck-Bogusz E, Kotylo P, Sliwinska-Kowalska M. Evaluation of voice acoustic parameters related to the vocal-loading test in professionally active teachers with dysphonia. Int J Occup Med Environ Health. 2007;20(1):25-30.

18. Cancian P, Passos GC, Martins EC, Perez F. Projeto Saúde Vocal do Professor: estratégias de intervenção em grupo - relato de experiência. In: Ferreira, LP, Silva MAA, organizadores. Saúde vocal: práticas fonoaudiológicas. São Paulo: Rocca; c2002. p.191-7.

19. Behlau M, Dragone MLS, Nagano L. A voz que ensina: o professor e a comunicação oral em sala de aula. Rio de Janeiro: Revinter; c2004.

20. Silverio KCA, Gonçalves CGO, Penteado RZ, Vieira TPG, Libardi A,
Rossi D. Ações em saúde vocal: proposta de melhoria do perfil vocal de professores. Pró-Fono. 2008;20(3):177-82.

21. Schneider B, Bigenzahn W. [Results of videostroboscopic examinations of vocally healthy candidates for voice intensive professions]. Laryngorhinootologie. 2002;8(12):894-9. German.

22. Frucht SJ, Fahn S, Greene PE, O'Brien C, Gelb M, Truong DD, et al. The natural history of embouchure dystonia. Mov Disord. 2001;16(5):899-906.

23. De Martini ML. Estudio comparativo de las caracteristicas de la respiracion en funcion fonica y la respiracion en funcion de la ejecucion de los instrumentos musicales de viento [tese]. Rosario: Facultad de Ciencias Medicas. Escuela de Fonoaudiogia; 2001. 115 p.

24. Simons DG, Travell JG, Simons LS. Travell \& Simons' myofascial pain and dysfunction: the trigger point manual. 2nd ed. Baltimore: Williams \& Wilkins; 1999. Vol. 1.

25. Lederman RJ. Neuromuscular and musculoskeletal problems in instrumental musicians. Muscle Nerve. 2003;27(5):549-61. Review. 